EUROPEAN ORGANIZATION FOR NUCLEAR RESEARCH

European Laboratory for Particle Physics

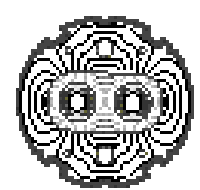

Large Hadron Collider Project

LHC Project Report 826

\title{
Emittance growth caused by electron cloud below the "fast TMCI" threshold: numerical noise or true physics?
}

\author{
$\underline{\text { E. Benedetto }}^{1)}$, G. Franchetti ${ }^{2)}$, K. Ohmi ${ }^{3)}$, D. Schulte, F. Zimmermann \\ CERN, Geneva, Switzerland
}

\begin{abstract}
Simulations show a persisting slow emittance growth for electron cloud densities below the threshold of the fast Transverse Mode Coupling type instability, which could prove important for proton beams with negligible radiation damping, such as in the LHC. We report on a variety of studies performed to quantify the contributions to the simulated emittance growth from numerical noise in the PIC module and from an artificial resonance excitation due to the finite number of kicks per turn applied for modeling the cloud-bunch interaction.
\end{abstract}

1) Politecnico di Torino, Turin, Italy

2) GSI, Darmstadt, Germany

3) KEK, Tsukuba, Japan

Presented at

PAC 2005, Knoxville, USA, May 16-20, 2005

CERN, CH-1211 Geneva 23,

Switzerland

Geneva, June 2005 


\title{
Emittance growth caused by electron cloud below the "fast TMCI" threshold: numerical noise or true physics?
}

\author{
E. Benedetto*, Politecnico di Torino, Italy, and CERN, Switzerland \\ D. Schulte, F. Zimmermann, CERN, Switzerland \\ G. Franchetti, GSI, Germany \\ K. Ohmi, KEK, Japan
}

\begin{abstract}
Simulations show a persisting slow emittance growth for electron cloud densities below the threshold of the fast Transverse Mode Coupling type instability, which could prove important for proton beams with negligible radiation damping, such as in the LHC. We report on a variety of studies performed to quantify the contributions to the simulated emittance growth from numerical noise in the PIC module and from an artificial resonance excitation due to the finite number of kicks per turn applied for modeling the cloud-bunch interaction.
\end{abstract}

\section{INTRODUCTION}

Electron cloud can cause beam blow up and emittance growth in proton and positron machines. If the electron density is higher than a certain 'threshold' value, a strong head-tail instability manifests itself, characterized by a large increase of the emittance in a time interval comparable to the synchrotron period. This phenomenon has been explained by a mechanism similar to the Transverse Mode Coupling Instability (TMCI) due to conventional impedances [1,2]. However, even below this 'TMCI' threshold, a long-term emittance growth has been seen in the simulations $[3,4,5]$. A series of studies investigated whether this slow beam size increase can be explained as a numerical artifact whether it represents true physics. That the electron cloud can induce emittance growth below the fast instability threshold is hinted at by observations of the vertical spot size as a function of beam current at the KEKB factory [6]. In this paper, we first present the results of pertinent simulations, where we identify several numerical parameters affecting the growth rate (number of macroparticles, number of kicks per turn,...). Then we discuss a possible explanation of the slow emittance growth below threshold as one caused by resonance crossing and modulational diffusion, or even loss of beam stability.

\section{SIMULATIONS TOOLS AND RESULTS}

The parameters of the simulations presented in this paper, if not otherwise mentioned, refer to the LHC at injection energy, and they are summarized in Table 1.

The code HEADTAIL [7] is used to study the single bunch transverse instabilities and emittance growth driven by the electron cloud. It computes the interaction between

\footnotetext{
* elena.benedetto@cern.ch
}

Table 1: Parameters used for LHC at injection

\begin{tabular}{lcc}
\hline electron cloud density & $\rho_{e}$ & $6 \times 10^{11} \mathrm{~m}^{-3}$ \\
bunch population & $N_{b}$ & $1.1 \times 10^{11}$ \\
beta function & $\beta_{x, y}$ & $66.0,71.54 \mathrm{~m}$ \\
rms bunch length & $\sigma_{z}$ & $0.13 \mathrm{~m}$ \\
rms beam size & $\sigma_{x, y}$ & $0.884 \mathrm{~mm}$ \\
rms momentum spread & $\delta_{\text {rms }}$ & $4.68 \times 10^{-4}$ \\
synchrotron tune & $Q_{s}$ & 0.0059 \\
momentum compaction & $\alpha_{c}$ & $3.47 \times 10^{-4}$ \\
circumference & $C$ & $26.659 \mathrm{~km}$ \\
nominal tunes & $Q_{x, y}$ & $64.28,59.31$ \\
chromaticity & $Q_{x, y}^{\prime}$ & 0,0 \\
relativistic factor & $\gamma$ & 479.6 \\
cavity voltage & $V$ & $8 \mathrm{MV}$ \\
harmonic number & $h$ & 35640 \\
\hline \# of macro-electrons & $N E L$ & $10^{5}$ \\
\# of macro-protons & $N P R$ & $3 \times 10^{5}$ \\
\# of slices & $N B I N$ & 70 \\
\# of grid points & $N$ & $128 \times 128$ \\
size of the grid & $\sigma_{g}$ & $10 \sigma_{x, y}$ \\
bunch extension in z & & $\pm 2 \sigma_{z}$ \\
\# of Interaction Points & $n k i c k$ & 1 \\
\hline
\end{tabular}

the beam and the electrons at a finite number of locations around the ring via a PIC module. The macro-particles representing the beam are transported between successive interaction points via a transfer matrix. The cloud evolves during the passage of the bunch, creating a transverse potential which depends on the longitudinal coordinate $z$, i.e., the longitudinal distance from the center of the bunch. At every interaction point, the electron distribution is 'refreshed' and the electric field is computed anew, according to the actual positions of the macroparticles.

In order to focus on the incoherent emittance growth and to speed up the simulations, we have introduced in the code the possibility to compute the potential created by the electrons only at the first interaction point and to use the same potential for the subsequent ones (weak-strong approximation). The electric field acting on the protons still depends on the longitudinal position of the particle, but the field stays the same for every turn and every interaction point. We call this new option the frozen-cloud or staticpotential approximation. However, the electron distribution still evolves during the passage of the bunch [8], or, in other words, the frozen potential is still $z$-dependent. Of course, the frozen field approximation cannot give rise to 
the TMCI-like instability, but for electron-cloud densities below the threshold of the TMC instability, the simulated emittance growth rate of a bunch moving in the static potential is about the same as that found with the standard 'strong-strong' HEADTAIL code, where the electron cloud evolves dynamically and the electric fields are recomputed at every interaction point; see Figs. 1 and 2.

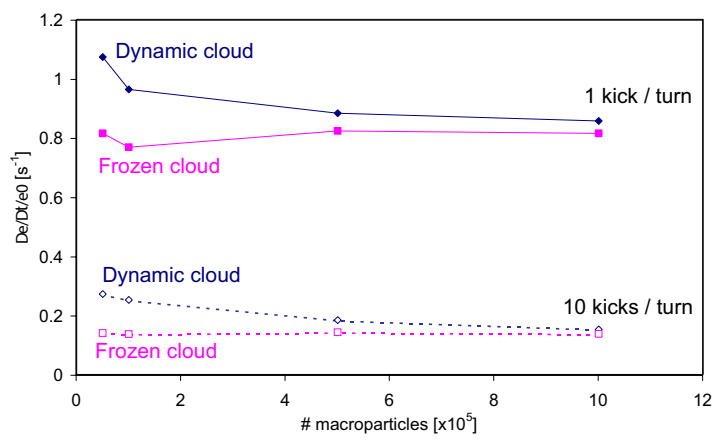

Figure 1: Emittance growth rate as a function of the number of macroparticles for electrons and protons, for 1 (top) and 10 interaction-points/turn (bottom lines); simulation results for dynamic (blue line) and frozen cloud (red) are compared.

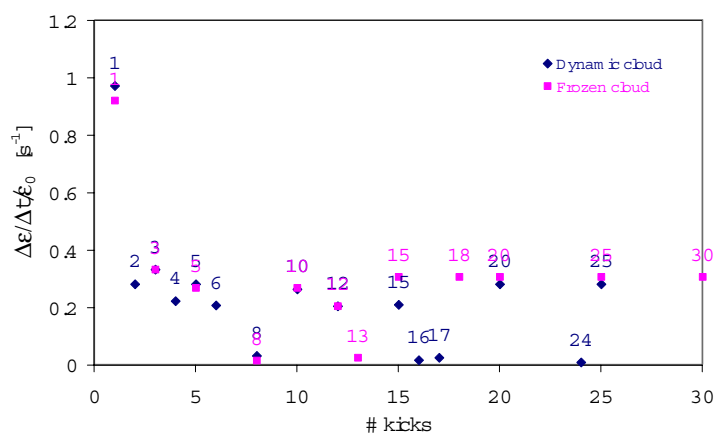

Figure 2: Emittance growth rate vs. the number of interaction points per turn.

Figure 1 presents the dependence of the emittance growth rate on the number of macroparticles. The weak dependence rules out the noise from the finite particle number as responsible for the growth. More importantly, in Fig. 1 the emittance growth drastically changes with the number of interactions per turn: For 10 such interactions the growth is about 4 or 5 times smaller than with only a single one. Unfortunately, the behaviour is not monotonic with an increasing number of kicks, as is illustrated in Fig. 2. This is due to the fact that when the number of interactions per turn is increased, we reduce the strength of the interactions, distributing it over several points around the ring, but at the same time we also change the effective phase advance between successive interaction points. This leads to excitation of different resonances in a non-monotonic way $[9,5]$, as is shown in Fig. 3.

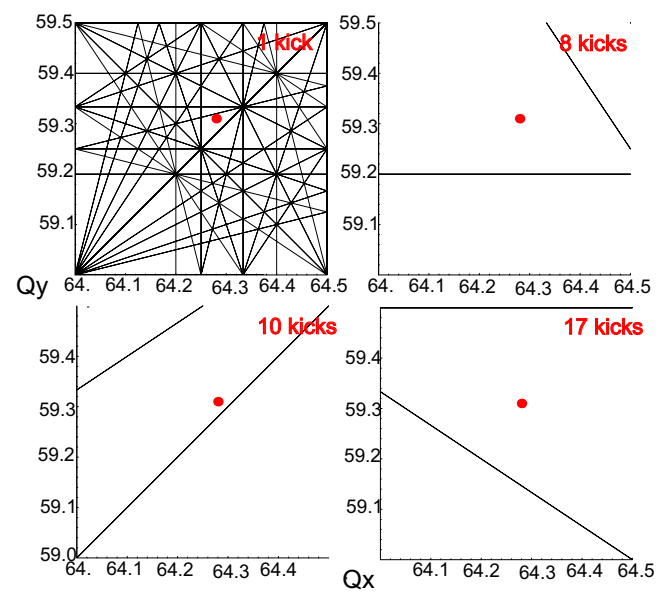

Figure 3: Tune diagram with simulated working point (red point), highlighting the resonance lines up to the 5 th order excited for different numbers of interaction points per turn: $1,8,10$, and 17 .

Other simulations demonstrate that the emittance growth rate does not depend on the initialization of the macroparticle position. In particular, the simulated growth rate is the same, whether the initial distribution of both protons and electrons is chosen axially symmetric or not. In addition, without synchrotron motion the simulated emittance growth quickly stops after a small initial blow up, which suggests an emittance-growth mechanism, described in the next section.

\section{RESONANCE CROSSING, HALO AND CORE EMITTANCE GROWTH}

A possible explanation to the slow term emittance growth can be found in analogy to emittance-growth processes in space-charge dominated beam [10]. The detuning induced by the space charge (which is negative) or by the electron cloud (positive) depends on the longitudinal position of the proton in the bunch. Particularly, for the electron cloud, there is no effect at the head of the bunch, while later on the electrons pinch towards the beam center, increasing the focusing. Therefore, protons performing synchrotron motion will experience a different tune shift, as they move from the head to the tail of the bunch. The main frequency of this modulation is the synchrotron frequency, while in the case of space charge it is twice this frequency. The periodic detuning may induce the periodic crossing of resonances, which can be excited either by lattice errors or by the non-linear component of the electron cloud itself. Also artificial resonances may be excited by the finite number of kicks in our simulation model, but a similar situation could arise in reality, if the electron cloud density varies along the ring or between beamline elements.

Figure 4 shows the horizontal invariant (action) of a single proton at a large synchrotron amplitude, as obtained from HEADTAIL. One can clearly see periodic jumps in 


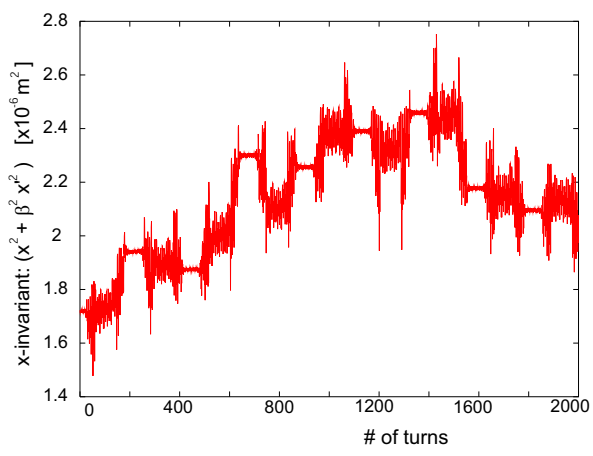

Figure 4: Horizontal action of a proton at a large synchrotron amplitude as a function of turn number, from a HEADTAIL simulation.

the action at the synchrotron frequency, characteristic of 'modulational diffusion'.

A simple toy code was written to study the dynamics of the protons in a constant focusing lattice, subjected to space charge or electron cloud forces. It assumes that the electron cloud kick strength increases linearly from the head to the tail of the bunch. The maximum tune shift, at the tail, is chosen as 0.04. The electron-cloud force on a test proton is:

$$
x^{\prime \prime}=K \frac{z-3 \sigma_{z}}{6 \sigma_{z}} \frac{x}{r^{2}}\left(1-e^{-\frac{r^{2}}{2 \sigma_{e}^{2}}}\right),
$$

here assuming that a longitudinal bunch edge at $3 \sigma_{z}$. In the above formula $\sigma_{e}$ is the rms size of the pinched electron cloud in the bunch, which is assumed to be Gaussian with $\sigma_{e}=\sigma_{\text {bunch }} / \sqrt{2}$ and the constant $K$ is the perveance generated by the electrons. Simulations using this toy model show that for this specific electron-cloud shape the emittance growth is mainly due to halo formation (Fig. 5). On the other hand, simulations with the HEADTAIL code in the frozen potential approximation exhibit a blow up of the beam core (also Fig. 5. This difference is attributed to the fact that the real cloud evolution is more complicated than that considered in the simple model: the electrons first pinch, producing a spiked and narrow distribution on the axis, which then relaxes and pinches again a second and third time during the passage of the bunch (see Fig. 6). The highly spiked distribution, combined with a finite number of kicks may even cause instability of the linear motion causing the core emittance growth.

\section{CONCLUSIONS}

The long-term emittance growth induced by an electron cloud below the 'TMCI' threshold appears to be real. Simulations suggest that it is caused by resonance crossing and modulational diffusion due to the combined effect of synchrotron motion and electron pinch. Predicting the exact growth rate requires understanding which resonances will be excited in the real machine. This can be determined by an accurate modelling of the lattice and the electron distribution [11].

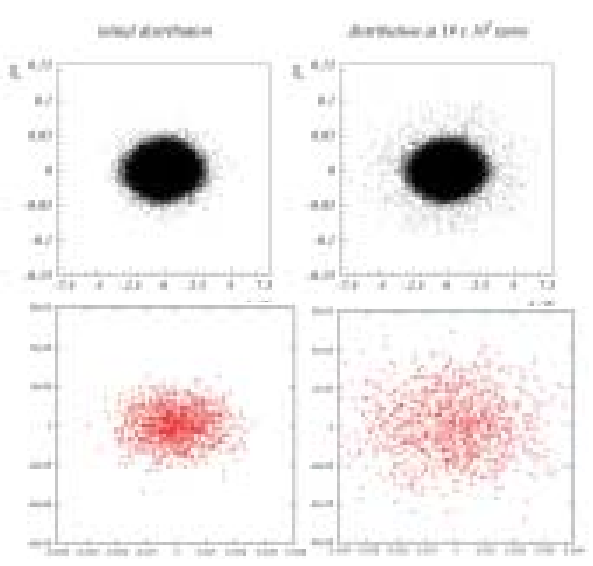

Figure 5: Initial and final $x-x^{\prime}$ phase space simulated by the 'toy model' (up)and by HEADTAIL (low)

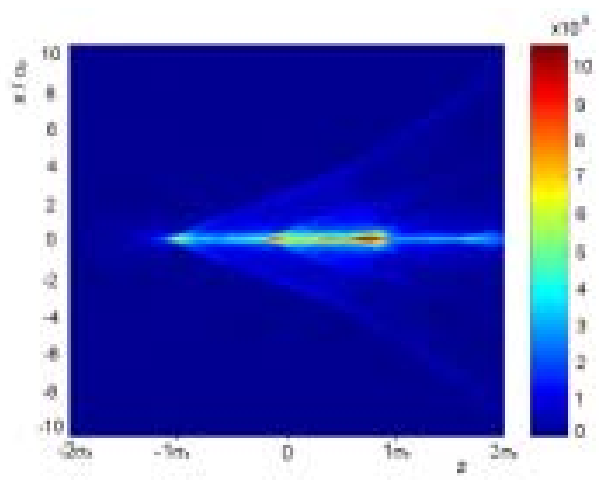

Figure 6: Electron density evolution during the passage of a bunch obtained from HEADTAIL.

\section{REFERENCES}

[1] K. Ohmi, F. Zimmermann, Phys. Rev. Letters 85, 3831 (2000)

[2] K. Ohmi, F. Zimmermann, E. Perevedentsev, Phys. Rev. E 65, 016502 (2002).

[3] E. Benedetto et al., Proc. EPAC'04, Lucerne, p. 1831 (2004).

[4] E. Benedetto et al., Proc. PAC'03, Portland, p. 3053 (2003).

[5] K. Ohmi, KEK Acc.-Physics Seminar March 2005.

[6] H. Fukuma, Proc. ECLOUD’04 Napa (2004)

[7] G. Rumolo, F. Zimmermann, "Practical User Guide for HEADTAIL," CERN-SL-Note-2002-036 (2002); see also same authors, PRST-AB 5:121002 (2002).

[8] E. Benedetto, F. Zimmermann, Proc. EPAC'04, Lucerne, p. 1834 (2004).

[9] E. Benedetto, F. Zimmermann, "Electron-Cloud Instability Simulations for the LHC," CARE HHH-2004 Workshop, CERN, (2004).

[10] G.Franchetti, "Space Charge and Optics Studies for HighIntensity Machine," CARE HHH-2004 Workshop, CERN, (2004).

[11] E. Benedetto et al., "Effect of Lattice...",these proceedings 